Individually adapted sequential Bayesian designs for conjoint choice experiments

J. Yu, P. Goos and M. Vandebroek

DEPARTMENT OF DECISION SCIENCES AND INFORMATION MANAGEMENT (KBI) 


\title{
Individually Adapted sequential Bayesian designs for conjoint choice experiments
}

\author{
Jie Yu \\ Faculty of Business and Economics, Katholieke Universiteit Leuven, Naamsestraat 69, \\ B-3000 Leuven, Belgium. \\ Email: Jie.Yu@econ.kuleuven.be \\ Tel: $+32(0) 16326962$ \\ Fax: +32 (0)163266 24
}

\section{Peter Goos}

Faculty of Applied Economics, Universiteit Antwerpen, Prinsstraat 13, B-2000 Antwerpen, Belgium.

Email: Peter.Goos@ua.ac.be

Tel: +32 (0)32204059

Fax: +32 (0)322048 17

\section{Martina Vandebroek}

Faculty of Business and Economics \& Leuven Statistics Research Centre, Katholieke Universiteit Leuven, Naamsestraat 69, B-3000 Leuven, Belgium.

Email: Martina.Vandebroek@econ.kuleuven.be

Tel: +32 (0)16326975

Fax: +32(0)163266 24 


\begin{abstract}
In this paper, we propose an efficient individually adapted sequential Bayesian approach for constructing conjoint choice experiments. It uses Bayesian updating, a Bayesian analysis and a Bayesian design criterion for generating choice-set-designs for each individual respondent based on previous answers of that particular respondent. The proposed design approach is compared with two non-adaptive design approaches (the average customization design proposed by Arora and Huber 2001 and the nearly orthogonal design constructed with Sawtooth software) under various degree of response error and respondent heterogeneity. The simulation study shows that the individually adapted sequential Bayesian approach leads to designs which are robust not only to respondent heterogeneity but also to response error. It turns out that the proposed method outperforms the benchmark methods in all scenarios that we have looked at. In particular, for conditions with high response error (the responses from a respondent can hardly provide proper information about the individual-level parameter and is therefore very challenging for individually adapted choice designs), our approach leads to substantially improvement not only in the precision of the parameter estimates but also in the predictive accuracy when the respondent heterogeneity is large. The new method therefore overcomes the limitation of the recently proposed adaptive polyhedral choice-based question design approach by Toubia et al. (2004), whose method performs well only when the response error is low. Furthermore, our study provides compelling evidence that adapting each respondent's choice sets based on the previous responses of that particular respondent in a Bayesian framework enables one to capture more information for the individuallevel parameters and therefore also on the population-level parameters. It is shown that it is substantially better to employ the adaptive approach when the response heterogeneity is high.
\end{abstract}

Keyword: adaptive Bayesian design, conjoint choice experiments, respondent heterogeneity, response error. 


\section{Introduction}

In marketing, conjoint choice experiments have frequently been carried out to assess consumer preferences for a certain product or service. In such an experiment, each respondent is requested to indicate his or her preferred product or service from each choice set presented to him or her. The data from such experiments are often analyzed by a random effects model which takes into account the heterogeneity in consumer preference by assuming that the partworths follow a distribution across consumers. In many situations, one is interested not only in the population-level preferences, but also in the individual-level preferences (Allenby and Rossi 1999; Arora et al. 1998; Arora and Huber 2001; Lenk et al. 1996; Revelt and Train 2000; Toubia et al. 2004; Train 2003). So far, Hierarchical Bayes modeling has been popular for estimating individual-level partworths. Individual preferences is obtained by combing the population-level distribution of the partworths and the information in each individual's choice data.

However, taking into account respondent preference heterogeneity poses a great challenge on the data collection because the data have to be good enough to capture the respondents' true behavioural variability in choice making (Hensher and Greene 2003). This implies that constructing an efficient design which ensures the data quality is very important. Sándor and Wedel (2002) and Yu et al. (2008a) show that the choice designs which take into account respondent heterogeneity do improve the precision of populationlevel parameter estimates and prediction.

In pioneering work on optimal design for choice experiments, the main focus has been on constructing one design that is assigned to all respondents (e.g. Burgess and Street 2003; Huber and Zwerina 1996; Kessels et al. 2006; Sándor and Wedel 2001, 2002; Vermeulen et al. 2008; Yu et al. 2008a, 2008b). The advantage of using different designs over the single design in terms of efficiency of parameter estimates has been demonstrated by Sándor and Wedel (2005), who constructed a full design which contains a limited set of different subdesigns that are offered to different respondents. These subdesigns are constructed in a non-adaptive way. That is, the choice sets are designed in advance and not based on the respondents' answers.

In the more general case, the choice designs for all respondents are different. For every respondent, each choice set in the experiment is constructed sequentially based on his or her previous responses. One of the challenges for the individually adapted sequential choice-set-design method is that the quality of the choice sets is influenced by the response errors in the previous responses (Hauser and Toubia 2005). Recently proposed adaptive methods by Toubia et al. (2003) and Toubia et al. (2004) tend to perform better than the non-adaptive benchmark methods when the response error is low, while they do not perform well when the response error is high. This implies that it is appealing to develop an 
individually adapted choice-set-design method which works well for low and high response error and is therefore robust to response error.

In this paper, we propose an individually adapted sequential Bayesian $(I A S B)$ approach for constructing choice designs. The main idea is to use the Bayesian update of the prior information and a Bayesian criterion for constructing the choice sets. More specifically, for every respondent, each choice set in the design is constructed using the Bayesian $D$-criterion with the prior information given by the posterior distribution based on all responses to previous choice sets by that respondent. This provides an efficient way to update the prior information that distinguishes the respondents. Compared to the non-adaptive Bayesian design approaches which construct designs in advance using a fixed prior (e.g. Huber and Zwerina 1996; Sándor and Wedel 2001; Arora and Huber 2001), the proposed adaptive approach allows one to update the prior repeatedly during the survey. This dynamic prior enables us to maximally utilize the available information from each respondent to construct the subsequent choice sets.

As opposed to Chaudhuri and Mykland (1993) who investigated only asymptotic properties, we consider small sample sizes and we generate choice sets for each respondent on the basis of his or her own prior responses, not on the responses of other respondents. Because each respondent ends up with a different single design with the prespecified number of choice sets, the cognitive burden is the same as when he or she receives a homogeneous design to evaluate. As the $I A S B$ design is tailor made for each respondent, it captures the individual-level preference better.

In the next section, we present the model used to capture respondent heterogeneity in discrete choice studies. In Section 3, we describe the construction of IASB designs. In Section 4, we evaluate our proposed approach for different response errors and respondent heterogeneity levels. Section 5 contains a summary of the main findings.

\section{Model Specification in Discrete Choice Study}

Suppose that a respondent has answered $S$ choice sets with $J$ alternatives per choice set. Let $\mathbf{Y}_{n}^{S}=\left(y_{n 1}, y_{n 2} \ldots, y_{n S}\right)$ denote the sequence of responses of respondent $n$ to these $S$ choice sets, and $\mathbf{X}_{n}^{S}$ denote all alternatives in all choice sets that have been assigned to respondent $n$. The probability to obtain the sequence of choices of respondent $n$ is

$$
P\left(\mathbf{Y}_{n}^{S} \mid \mathbf{X}_{n}^{S}, \boldsymbol{\beta}_{n}\right)=\prod_{s=1}^{S} p_{n s}\left(y_{n s} \mid \boldsymbol{\beta}_{n}\right)
$$


where

$$
p_{n s}\left(y_{n s} \mid \boldsymbol{\beta}_{n}\right)=\frac{\exp \left(\boldsymbol{\beta}_{n}^{\prime} \mathbf{x}_{n y_{n s} s}\right)}{\sum_{j=1}^{J} \exp \left(\boldsymbol{\beta}_{\boldsymbol{n}}^{\prime} \mathbf{x}_{n j s}\right)}
$$

with $p_{n s}\left(y_{n s} \mid \boldsymbol{\beta}_{n}\right)$ the choice probability of respondent $n$ in choice set $s, \mathbf{x}_{n y_{n s} s}$ characterizes the alternative chosen by respondent $n$ in choice set $s, \mathbf{x}_{n j s}$ characterizes alternative $j$ in choice set $s$ for respondent $n$, and $\boldsymbol{\beta}_{n}$ is a $p$-dimensional coefficient vector containing the effects of different attribute levels on the utility for respondent $n$. Since $\boldsymbol{\beta}_{n}$ is unknown, the probability of sequential choices of respondent $n$ not conditional on $\boldsymbol{\beta}_{n}$ is the integral of $P\left(\mathbf{Y}_{n}^{S} \mid \mathbf{X}_{n}^{S}, \boldsymbol{\beta}_{n}\right)$ over the distribution of $\boldsymbol{\beta}_{n}, g\left(\boldsymbol{\beta}_{n} \mid \boldsymbol{\mu}, \boldsymbol{\Sigma}\right)$ :

$$
\begin{aligned}
\pi\left(\mathbf{Y}_{n}^{S} \mid \mathbf{X}_{n}^{S}, \boldsymbol{\mu}, \boldsymbol{\Sigma}\right) & =\int P\left(\mathbf{Y}_{n}^{S} \mid \mathbf{X}_{n}^{S}, \boldsymbol{\beta}_{n}\right) g\left(\boldsymbol{\beta}_{n} \mid \boldsymbol{\mu}, \boldsymbol{\Sigma}\right) d \boldsymbol{\beta}_{n} \\
& =\int\left(\prod_{s=1}^{S} p_{n s}\left(y_{n s} \mid \boldsymbol{\beta}_{n}\right)\right) g\left(\boldsymbol{\beta}_{n} \mid \boldsymbol{\mu}, \boldsymbol{\Sigma}\right) d \boldsymbol{\beta}_{n}
\end{aligned}
$$

The model defined above is called the panel mixed logit model. A special case of this model is the cross-sectional mixed logit model investigated in the marketing literature by Sándor and Wedel (2002) and Yu et al. (2008). This model assumes that the choice probabilities for a single respondent are independent across choice sets. Under this assumption, the panel mixed logit model in (3) is simplified to

$$
\pi\left(\mathbf{Y}_{n}^{S} \mid \mathbf{X}_{n}^{S}, \boldsymbol{\mu}, \boldsymbol{\Sigma}\right)=\prod_{s=1}^{S} \int p_{n s}\left(y_{n s} \mid \boldsymbol{\beta}_{n}\right) g\left(\boldsymbol{\beta}_{n} \mid \boldsymbol{\mu}, \boldsymbol{\Sigma}\right) d \boldsymbol{\beta}_{n}
$$

The model in (4) is called the cross-sectional mixed logit model. Comparing expressions (3) and (4), we see that the panel mixed logit model which takes into account the within respondent correlation across repeated choice observations is more realistic since the observations from any particular respondent tend to have the same underlying behavior.

In this paper, we consider the panel mixed logit model. The corresponding likelihood function of the observed responses for $N$ respondents is

$$
\begin{aligned}
L\left(\mathbf{Y}_{N} \mid \mathbf{X}_{N}, \boldsymbol{\mu}, \boldsymbol{\Sigma}\right) & =\prod_{n=1}^{N} \pi\left(\mathbf{Y}_{n}^{S} \mid \mathbf{X}_{n}^{S}, \boldsymbol{\mu}, \boldsymbol{\Sigma}\right), \\
& =\prod_{n=1}^{N} \int\left(\prod_{s=1}^{S} p_{n s}\left(y_{n s} \mid \boldsymbol{\beta}_{n}\right)\right) g\left(\boldsymbol{\beta}_{n} \mid \boldsymbol{\mu}, \boldsymbol{\Sigma}\right) d \boldsymbol{\beta}_{n},
\end{aligned}
$$

where $\mathbf{Y}_{N}$ contains the responses for $N$ respondents and $\mathbf{X}_{N}$ is the full design matrix which contains the subdesigns for $N$ respondents.

We use a Hierarchical Bayes approach to estimate the panel mixed logit model. The 
advantage of this approach is that it can provide the estimates for individual-level parameters by combining the information from each individual's choice data and the distribution of population preferences.

\section{Constructing Individually Adapted Sequential Bayesian Designs $(I A S B)$}

As mentioned in the introduction, Sándor and Wedel (2002) construct efficient locally $D$ optimal designs for the cross-sectional mixed logit model. Yu et al. (2008a) extend their locally optimal design procedure to obtain Bayesian optimal designs using an efficient algorithm. Both studies take into account the respondent heterogeneity in the design construction by using a design criterion based on the Fisher information matrix derived from the cross-sectional mixed logit model. However, constructing designs for cross-sectional mixed logit models is not an easy task since the Fisher information matrix involves many numerical integrations over the distribution of the random model coefficients. Unfortunately, the construction of the choice designs for panel mixed logit model is far more complex than the cross-sectional mixed logit model because the Fisher information matrix for the former cannot be determined independently of the responses. This significantly adds to the complexity of the design process.

The individually adapted sequential Bayesian $(I A S B)$ approach introduced in this section provides an alternative solution to construct choice designs which take into account respondent heterogeneity. Compared to the mixed logit design approach, the $I A S B$ approach is much easier to implement. The idea is to generate each respondent's choice sets in a Bayesian framework based on the previous responses given by that respondent. At the end of the survey, each respondent ends up with a different design that is tailored to the parameters for that respondent. If there are $N$ respondents, then the full design actually consists of $N$ subdesigns, where each subdesign is tailor made for each participant. As a result, the individual heterogeneity is taken into account in the full design. This is different from the design approaches used by Arora and Huber (2001), Burgess and Street (2003), Huber and Zwerina(1996), Kessels et al. (2006), Sándor and Wedel (2001), Vermeulen et al. (2008), whose full designs consist of $N$ homogeneous designs. The homogeneous design is constructed in a way which is optimal for the average respondent. Such a design is referred to as aggregate customization design. To better understand the design construction, we first review how to generate the choice designs in general.

The Bayesian design criterion that we used is based on the Generalized Fisher Information matrix (see $\mathrm{Yu}$ et al. 2008b for a detailed explanation), which is obtained by taking the negative expectation of the second derivative of the log-posterior density of the model parameters, $\mathcal{I}_{G F I M}(\boldsymbol{\beta}, \mathbf{X})=-\mathrm{E}\left[\partial^{2} \log h(\boldsymbol{\beta} \mid \mathbf{Y}, \mathbf{X}) /\left(\partial \boldsymbol{\beta} \partial \boldsymbol{\beta}^{\prime}\right)\right]$, with $h(\boldsymbol{\beta} \mid \mathbf{Y}, \mathbf{X})$, the 
posterior distribution corresponding to a design $\mathbf{X}$ and responses $\mathbf{Y}$. A one-dimensional measure of the efficiency of design $\mathbf{X}$ is the $D$-error $(\boldsymbol{\beta}, \mathbf{X})=\operatorname{det}\left[\mathcal{I}_{G F I M}(\boldsymbol{\beta}, \mathbf{X})\right]^{-1 / p}$ with $p$ the dimension of the parameter of vector $\boldsymbol{\beta}$. The expectation of the $D$-error over the prior distribution of the parameter values, $\pi(\boldsymbol{\beta})$, is denoted by the $D_{B}$-error $(\mathbf{X})=$ $\int \operatorname{det}\left[\mathcal{I}_{G F I M}(\boldsymbol{\beta}, \mathbf{X})\right]^{-1 / p} \pi(\boldsymbol{\beta}) d \boldsymbol{\beta}$. The design which minimizes the $D_{B}$-error $(\mathbf{X})$ is considered as the best design. A critical issue in constructing efficient designs is to find out the best source for determining the prior information before constructing the design. In our approach, we update the prior information repeatedly using Bayesian methods.

The proposed design procedure consists of two stages: an initial static stage followed by a fully adaptive sequential stage. Let $g_{0}\left(\boldsymbol{\beta}_{n}\right)$ be the initial prior which is available before conducting the experiment for respondent $n$. This prior will be used to conduct the Bayesian analysis once the responses of respondent $n$ from the static stage are observed. Note that we do not assume that different initial priors for the different participants are available. In practice, it is possible to get some idea about the population parameters using either historical data or pilot studies. It is not common to let the same participants be involved in both the pilot study and the ongoing survey as they will get tired. Therefore, individual-level information is usually not available in the initial stage. In our study, we use a common initial prior for all respondents reflecting the prior information on the population heterogeneity. The disadvantage of using this prior is that for respondents whose true parameters are far from the population mean, this common prior will provide misleading information on the individual-level parameters. This is particularly true when the respondent heterogeneity is high. Fortunately, the adaptive sequential approach enables one to update the initial prior repeatedly during the sequential design process. The results from the simulation study show that this approach works quite well in a broad range of scenarios.

In the static stage, we choose $s_{1}$ choice sets randomly for each respondent. Suppose that the responses of respondent $n, \mathbf{Y}_{n}^{s 1}$, corresponding to the $s_{1}$ initial choice sets, $\mathbf{X}_{n}^{s_{1}}$, are observed after the initial experiment is carried out. The posterior distribution based on $\mathbf{X}_{n}^{s_{1}}, \mathbf{Y}_{n}^{s 1}$ and the prior $g_{0}\left(\boldsymbol{\beta}_{n}\right)$ for respondent $n$ can be computed as follows

$$
h\left(\boldsymbol{\beta}_{n} \mid \mathbf{Y}_{n}^{s_{1}}, \mathbf{X}_{n}^{s_{1}}\right)=\frac{L\left(\mathbf{Y}_{n}^{s_{1}} \mid \mathbf{X}_{n}^{s_{1}}, \boldsymbol{\beta}_{n}\right) g_{0}\left(\boldsymbol{\beta}_{n}\right)}{\int L\left(\mathbf{Y}_{n}^{s_{1}} \mid \mathbf{X}_{n}^{s_{1}}, \boldsymbol{\beta}_{n}\right) g_{0}\left(\boldsymbol{\beta}_{n}\right) d \boldsymbol{\beta}_{n}}
$$

We consider this posterior distribution $h\left(\boldsymbol{\beta}_{n} \mid \mathbf{Y}_{n}^{s_{1}}, \mathbf{X}_{n}^{s_{1}}\right)$ as the prior for constructing the $\left(s_{1}+\right.$ $1)$ th choice set, $\mathbf{x}_{n}^{s_{1}+1}$. The choice set $\mathbf{x}_{n}^{s_{1}+1}$ is chosen such that the $D_{B}$-error $\left(\mathbf{X}_{n}^{s_{1}}, \mathbf{x}_{n}^{s_{1}+1}\right)$ is minimized. To compute the $D_{B}$-error $\left(\mathbf{X}_{n}^{s_{1}}, \mathbf{x}_{n}^{s_{1}+1}\right)$, we have to take a large number of draws from the updated prior distribution, $h\left(\boldsymbol{\beta}_{n} \mid \mathbf{Y}_{n}^{s_{1}}, \mathbf{X}_{n}^{s_{1}}\right)$. Unfortunately, taking draws from $h\left(\boldsymbol{\beta}_{n} \mid \mathbf{Y}_{n}^{s_{1}}, \mathbf{X}_{n}^{s_{1}}\right)$ is usually intractable since $h\left(\boldsymbol{\beta}_{n} \mid \mathbf{Y}_{n}^{s_{1}}, \mathbf{X}_{n}^{s_{1}}\right)$ has no closed form. One solution is to use a discrete approximation to $h\left(\boldsymbol{\beta}_{n} \mid \mathbf{Y}_{n}^{s_{1}}, \mathbf{X}_{n}^{s_{1}}\right)$, such as importance sampling discussed by Bedrick et al. (1997), Monahan and Genz (1997), Rossi et al. (2005), Train 
(2003). In this paper, we employ this discrete approximation approach too. The method can be described as follows.

1. Compute the mode, $\boldsymbol{\beta}_{n}^{*}$, and the Hessian matrix, $\mathcal{H}$, of $h\left(\boldsymbol{\beta}_{n} \mid \mathbf{Y}_{n}^{s_{1}}, \mathbf{X}_{n}^{s_{1}}\right)$ distribution.

2. Take $R$ draws denoted as $\boldsymbol{\beta}_{n}^{r}$ from the importance density, which is the multivariate student t with $\boldsymbol{\beta}_{n}^{*},-\mathcal{H}^{-1}$ and $v$ as the parameters, where $v$ is the degree of freedom.

3. Compute the corresponding weight $W^{r}$ for each $\boldsymbol{\beta}_{\boldsymbol{n}}^{r}$. Suppose that $h^{*}\left(\boldsymbol{\beta}_{n} \mid \mathbf{Y}_{n}^{s_{1}}, \mathbf{X}_{n}^{s_{1}}\right)$ is the density kernel of $h\left(\boldsymbol{\beta}_{n} \mid \mathbf{Y}_{n}^{s_{1}}, \mathbf{X}_{n}^{s_{1}}\right)$ and $f\left(\boldsymbol{\beta}_{n}\right)$ is the kernel of the importance density, the weight $W^{r}$ for $r$ th draw, $\boldsymbol{\beta}_{n}^{r}$, is computed as

$$
W^{r}=\frac{h^{*}\left(\boldsymbol{\beta}_{n}^{r} \mid \mathbf{Y}_{n}^{s_{1}}, \mathbf{X}_{n}^{s_{1}}\right) / f\left(\boldsymbol{\beta}_{n}^{r}\right)}{\sum_{r=1}^{R} h^{*}\left(\boldsymbol{\beta}_{n}^{r} \mid \mathbf{Y}_{n}^{s_{1}}, \mathbf{X}_{n}^{s_{1}}\right) / f\left(\boldsymbol{\beta}_{n}^{r}\right)}
$$

4. The $D_{B^{-}} \operatorname{error}\left(\mathbf{X}_{n}^{s_{1}}, \mathbf{x}_{n}^{s_{1}+1}\right)$ is approximated by

$$
D_{B^{-}} \operatorname{error}\left(\mathbf{X}_{n}^{s_{1}}, \mathbf{x}_{n}^{s_{1}+1}\right)=\sum_{r=1}^{R} D-\operatorname{error}\left(\mathbf{X}_{n}^{s_{1}}, \mathbf{x}_{n}^{s_{1}+1}, \boldsymbol{\beta}_{n}^{r}\right) W^{r} .
$$

We consider the $\mathbf{x}_{n}^{s_{1}+1}$ which minimizes the $D_{B}$-error $\left(\mathbf{X}_{n}^{s_{1}}, \mathbf{x}_{n}^{s_{1}+1}\right)$ as the final design for the $\left(s_{1}+1\right)$ th choice set. This choice set is then assigned to respondent $n$ and the $\left(s_{1}+\right.$ 1)th observation is obtained. We then update the prior information based on all $s_{1}+1$ observations and construct the $\left(s_{1}+2\right)$ th choice set for that respondent. All further choice sets are generated similarly until a prespecified number $S$ is reached. As such, every respondent ends up with a different design that is tailored to the parameters for that respondent. It is expected that the individually adapted sequential Bayesian approach enables us to obtain more information on the individual-level parameters than existing non-adaptive procedures.

\section{Simulation study}

In this section, we investigate the performance of the $I A S B$ approach in terms of estimation accuracy and prediction capability. We investigate different levels of response error because this strongly affects the results of conjoint choice studies. With high response errors, the responses from a respondent can hardly provide proper information about the individual-level parameter and is therefore very challenging for individually adapted choice designs.

To study whether the $I A S B$ approach for constructing choice-based designs is robust to response error, we adopt the simulation structure proposed by Arora and Huber (2001) for hierarchical Bayes estimation and also utilized by Toubia et al. (2004) in the context of 
choice experiments. They varied both the response accuracy and consumer heterogeneity by selecting a low and a high level. We consider designs with three attributes, each at three levels. Following Arora and Huber (2001) and Toubia et al. (2004), we choose the partworths for the population mean corresponding to each attribute evenly spaced between $[-a r]$ with $a=0.5$ in case of low response accuracy and $a=3$ in case of high response accuracy. Note that larger magnitudes of $a$ lead to higher response accuracy because the variance of the Gumbel distribution is inversely proportional to the squared magnitude of the partworths (Ben-Akia and Lerman 1985). Arora and Huber (2001) and Toubia et al. (2004) model respondent heterogeneity by assuming that the individual-level parameters vary according to a multivariate normal distribution with mean $\boldsymbol{\mu}$ and covariance matrix $\sigma_{\boldsymbol{\beta}}^{2} \mathbf{I}_{p}$. Note that we call $\sigma_{\boldsymbol{\beta}}$ the heterogeneity parameter and assume it is constant across all attribute levels. The way we define the degree of heterogeneity is the same as in Arora and Huber (2001) and Toubia et al. (2004). In case of high respondent heterogeneity, the value of $\sigma_{\boldsymbol{\beta}}$ is given by $\sigma_{\boldsymbol{\beta}}^{2} / a=3$, and in case of low respondent heterogeneity, the value of

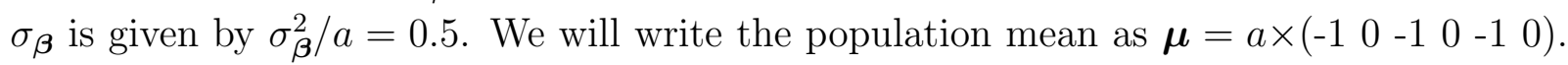

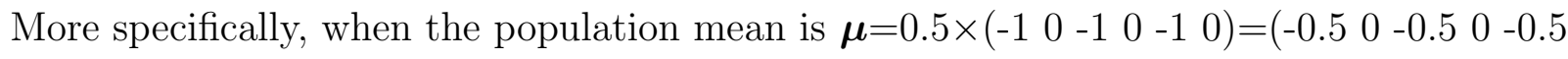
$0)$, the corresponding $\sigma_{\boldsymbol{\beta}}^{2}$ equals 0.25 to express low heterogeneity and 1.5 to express high heterogeneity. When $\boldsymbol{\mu}=\left(\begin{array}{lllll}-3 & 0 & -3 & 0 & -3\end{array}\right)$, the corresponding $\sigma_{\boldsymbol{\beta}}^{2}$ equals to 1.5 for low and 9 for high heterogeneity in the population.

Arora and Huber (2001) proposed aggregate customization as an approach to improve individual-level estimates using a hierarchical Bayes choice model. The idea is to use prior estimates to build a common design customized for the average respondent. They compared the performance of the customized design to that of an orthogonal design in terms of the accuracy of the individual-level parameter estimates and prediction capabilities using the simulation structure described above.

Toubia et al. (2004) proposed a polyhedral method for constructing choice-based question design. They compared their approach to an orthogonal design, a random design and the customized design proposed by Arora and Huber (2001) using the same simulation structure as Arora and Huber (2001). They found that their method is quite sensitive to the response error. It performs well only in case of the high response accuracy (e. g., for large values of $a$ ). However, when the response error is high, the negative effects from the high response error dominate the benefits from the individual adaptation, and the polyhedral question design does not work well.

To be consistent with the prior studies on adaptive choice designs, we also consider the customized design proposed by Arora and Huber (2001) and the orthogonal design as benchmarks. In the simulation study, we assume that the total number of choice sets that a respondent has to evaluate is $S=16$, with three alternatives per choice set. The first 
five choice sets form the initial static stage, the following eleven choice sets are constructed sequentially. We construct a nearly orthogonal design using the Sawtooth software, because such a design is commonly used by researchers who do not have access to advanced methodology for computing optimal designs for conjoint choice studies. As in Arora and Huber (2001) and Toubia et al. (2004), we assume that pretest data are available. Based on these pretest data, prior information on the population mean and variance can be obtained. We use this information as the input for constructing the customized design as Arora and Huber (2001) and Toubia et al. (2004) did. We assume that the number of respondents who participate in the experiment is 250. For each approach, a Hierarchical Bayes model is estimated by using $M C M C$ which combines the Gibbs sampler with the Metropolis-Hastings method.

We consider two criteria to assess the precision of the parameter estimates for each approach. Both criteria are based on the root mean square error $\left(R M S E_{\boldsymbol{\beta}}\right)$ of the estimated parameters of an individual. The $R M S E_{\boldsymbol{\beta}}$ assesses how far the estimated individual-level parameters are from the true parameters and has also been used in the studies of Arora and Huber (2001) and Toubia et al. (2004). To quantify the extent to which the IASB approach performs better than the two benchmark approaches in terms of the accuracy of the parameter estimates, we compute the percentage decrease in $R M S E$ by using the $I A S B$ approach over each of the benchmark approaches for each respondent. We average these percentage values over all respondents in the study. These averaged values are given in Table 1 . The row labeled $I A S B / O R G$ shows the averaged percentage decrease in $R M S E_{\boldsymbol{\beta}}$ by using the $I A S B$ approach instead of the nearly orthogonal design approach. Similarly, the row labeled $I A S B / C U M$ presents us how much better the $I A S B$ approach is compared to the customized approach. In addition, Table 1 also presents the percentage of the respondents for whom the design method yields the lowest $R M S E_{\boldsymbol{\beta}}$.

We also use two criteria to measure the prediction capability. The hit rate is defined as the percentage of times that an approach correctly predicts the most-preferred alternative. To compute the hit rate, we use the complete choice design proposed by Kessels et al. (2006) as the holdout design. This design consists of all possible choice sets. For the design problem that we considered, three attributes with three levels per attribute and three alternatives per choice set, the complete choice design consists of 2925 choice sets. In addition, we are interested in the percentage decrease in the root mean square prediction error $\left(R M S E_{p}\right)$ by using one design method over another. The row labeled $I A S B / O R G$ in Table 2 indicates the improvement in predictive accuracy using the $I A S B$ approach instead of the nearly orthogonal approach, while the row labeled $I A S B / C U M$ has similar interpretation but compared with the customized design.

Several conclusions can be drawn from Table 1 and Table 2 in terms of both parame- 
Table 1: Robustness of each design method to response accuracy and the heterogeneity in terms of estimation

\begin{tabular}{|c|l|c|c|c|c|}
\hline Various Scenarios & $\begin{array}{l}\text { Response accuracy } \\
\text { Heterogeneity }\left(\sigma^{2}\right)\end{array}$ & $\begin{array}{c}\text { Low (0.5) } \\
\text { Low (0.25) }\end{array}$ & $\begin{array}{c}\text { Low (0.5) } \\
\text { High (1.5) }\end{array}$ & $\begin{array}{c}\text { High (3.0) } \\
\text { Low (1.5) }\end{array}$ & $\begin{array}{c}\text { High(3.0) } \\
\text { High (9.0) }\end{array}$ \\
\hline \multirow{2}{*}{ Decrease in RMSE } & IASB/ORG & $3.7 \%$ & $13.6 \%$ & $21.1 \%$ & $33.4 \%$ \\
& $I A S B / C U M$ & $0.1 \%$ & $18.7 \%$ & $11.9 \%$ & $31.6 \%$ \\
\hline \multirow{2}{*}{ Percentage Best } & Orthogonal & $30.8 \%$ & $28.7 \%$ & $17.5 \%$ & $10.4 \%$ \\
& Customized & $33.7 \%$ & $19.6 \%$ & $33.3 \%$ & $12.1 \%$ \\
& Sequential & $35.5 \%$ & $51.7 \%$ & $49.2 \%$ & $77.5 \%$ \\
\hline
\end{tabular}

Table 2: Robustness of each design method to response accuracy and the heterogeneity in terms of prediction

\begin{tabular}{|c|l|c|c|c|c|}
\hline Various Scenarios & $\begin{array}{l}\text { Response accuracy) } \\
\text { Heterogeneity }\left(\sigma^{2}\right)\end{array}$ & $\begin{array}{c}\text { Low (0.5) } \\
\text { Low (0.25) }\end{array}$ & $\begin{array}{c}\text { Low (0.5) } \\
\text { High (1.5) }\end{array}$ & $\begin{array}{c}\text { High (3.0) } \\
\text { Low (1.5) }\end{array}$ & $\begin{array}{c}\text { High(3.0) } \\
\text { High (9.0) }\end{array}$ \\
\hline \multirow{2}{*}{$\begin{array}{c}\text { Decrease in Prediction } \\
\text { Error }\end{array}$} & IASB/ORG & $5.87 \%$ & $16.8 \%$ & $32.1 \%$ & $48.5 \%$ \\
& $I A S B / C U M$ & $0.94 \%$ & $25.3 \%$ & $20.6 \%$ & $55.3 \%$ \\
\hline \multirow{2}{*}{ Hit rate } & Orthogonal & $76.9 \%$ & $82.5 \%$ & $89.2 \%$ & $86.9 \%$ \\
& Customized & $77.2 \%$ & $79.5 \%$ & $91.2 \%$ & $84.5 \%$ \\
& Sequential & $78.1 \%$ & $86.0 \%$ & $93.1 \%$ & $93.8 \%$ \\
\hline
\end{tabular}

ter estimation and prediction. First of all, for all scenarios and all evaluation criteria we looked at, the $I A S B$ design approach outperforms the customized design and the orthogonal design approaches. In particular, for scenarios where the response accuracy is high, our proposed approach performs substantially better than the non-adaptive benchmark approaches. Table 1 shows us that, compared to the nearly orthogonal design and the customized design approaches, the $I A S B$ design approach leads to a decrease in $R M S E$ of $21.1 \%$ up to $33.4 \%$ and $11.9 \%$ up to $31.6 \%$, respectively. Table 2 indicates that the decrease in prediction error is also remarkable, with the decreases of $32.1 \%$ up to $48.5 \%$ and $20.6 \%$ up to $55.3 \%$, respectively. This excellent performance is not unexpected since under these scenarios, the design of the subsequent choice sets in the adaptive sequential approach is based on more accurate information from previous responses.

Secondly, for any given level of response accuracy, the advantage of using the $I A S B$ approach over the two non-adaptive approaches increases with the consumer heterogeneity. Furthermore, for scenarios with high respondent heterogeneity (Column 4 and 6), the $I A S B$ approach yields a substantial improvement in efficiency over the benchmark approaches not only in terms of the parameter estimates but also in terms of the predictive accuracy. This implies that the proposed approach enables us to better capture the differences in individual preferences and obtain more information on the individual-level 
parameter estimates

The evaluation under the scenarios where the response accuracy is low $(a=0.5)$ are especially interesting as these are the scenarios where you can hardly get a reliable answer to the choice set presented to an individual respondent. Toubia et al. (2004) proposed a polyhedral method for updating the choice-set design sequentially for each individual. They found that these scenarios do not favor the individual-level adaptation. The response errors counteract the potential gains in choice-set selection due to individual-level adaptation. Surprisingly, the simulation results in Table 1 and Table 2 clearly demonstrate that even with the low response accuracy, the $I A S B$ approach still outperforms the other two approaches. In particular, we found that as long as the consumer heterogeneity is high $\left(\sigma^{2} / a=3\right)$, the advantage of the $I A S B$ approach is remarkable irrespective of the response error. This indicates that our proposed approach is more appealing in constructing adaptive choice design than the method proposed by Toubia et al. (2004), who found that with low response accuracy and high heterogeneity, the disadvantages of high response error appear to offset the need for individually adapted sequential design.

Finally, the comparison between the nearly orthogonal design and the customized design appears to be consistent with Arora and Huber (2001), who concluded that the customized design performs better than the orthogonal design approach when the magnitude of the population mean is high and the heterogeneity is low.

\section{Conclusion}

In this paper, we proposed an individually adapted sequential Bayesian design approach for constructing conjoint choice experiments. We compare the proposed approach with two non-adaptive benchmark design approaches (the average customization design proposed by Arora and Huber 2001 and the nearly orthogonal design constructed with Sawtooth software) under various degrees of response error and respondent heterogeneity. In the simulation study, we pay special attention to the scenarios where the response error is high as the quality of the choice-set-design is influenced by response errors in previous responses. The simulation study shows that the proposed $I A S B$ approach leads to designs which are robust not only to respondent heterogeneity but also to response error. It turns out that the $I A S B$ design by far outperforms the benchmark designs in all the scenarios that we have looked at. In particular, with low response accuracy and high heterogeneity, the $I A S B$ approach leads to substantially improvement not only in the precision of the parameter estimates but also in the predictive accuracy. This overcomes the limitations of the recently proposed adaptive polyhedral choice-based question design approach by Toubia et al. (2004), whose method only performs well when the response error is low. They concluded that in case of low response accuracy and high heterogeneity, the impre- 
cise answers appear to offset the need for individually adapted designs. Furthermore, our study provides very strong evidence that generating each respondent's choice sets based on previous answers of that particular respondent in a Bayesian framework enables us to capture more information on the individual-level parameter estimates than the benchmark approaches. In case the response heterogeneity is high, the gains in precision in both parameter estimates and prediction are remarkably large.

\section{Acknowledgments}

We are grateful to Zsolt Sándor for his helpful suggestions and contributions to this paper.

In addition, we acknowledge the financial support of the research council of the Katholieke Universiteit Leuven (OT-project 04/07).

\section{References}

Allenby, G. M., P. E. Rossi. 1999. Marketing models of consumer heterogeneity. Journal of Econometrics. 89 57-78.

Arora, N., G. M. Allenby, J. L. Ginter. 1998. A Hierarchical Bayes model of primary and secondary demand. Marketing Science. 17 29-44.

Arora, N., J. Huber. 2001. Improving parameter estimates and model prediction by aggregate customization in choice experiments. Journal of Consumer Research. 28 273-283.

Bedrick, E. J., R. Christensen, W. JohnsonBayesian. 1997. Binomial Regression: Predicting Survival at a Trauma Center. The American Statistician. 51 211-218.

Ben-Akiva, M., S. R. Lerman (1985). Discrete choice analysis: theory and application to travel demand. Cambridge: Massachusetts Institute of Technology Press.

Burgess, L., D. J. Street. 2003. Optimal designs for $2^{k}$ choice experiments. Communications in Statistics, Theory and Methods. 32 2185-2206.

Chaudhuri, P., P. A. Mykland. 1995. On efficient designing of nonlinear experiments. Statistica Sinica. 5 421-440.

Hauser, J. R., O. Toubia. 2005. The impact of endogeneity and utility balance in conjoint analysis. Marketing Science. 24 498-507. 
Hensher, D. A., W. H. Greene. 2003. The mixed logit model: The state of practice. Transportation 30 133-176.

Huber, J., K. Zwerina. 1996. The importance of utility balance in efficient choice designs. Journal of Marketing Research. 307-317.

Kessels, R., P. Goos, M. Vandebroek. 2006. A comparison of criteria to design efficient choice experiments. Journal of Marketing Research. 43 409-419.

Lenk, P. J., W. S. DeSarbo, P. E. Green, M. R. Young. 1996. Hierarchical Bayes conjoint analysis: Recovery of partworth heterogeneity from reduced experimental designs. Marketing Science. 15 173-191.

Monahan, J., A. Genz. 1997. Spherical-Radial Integration Rules for Bayesian Computation. Journal of the American Statistical Association. 92 664-674.

Revelt, D., K. Train. 2000. Customer-Specific Taste Parameters and Mixed Logit: Households' Choice of Electricity Supplier. Working paper.

Rossi, P. E., G. M. Allenby, R. McCulloch. 2005. Bayesian statistics and marketing. Wiley series in probability and statistics. Wiley.

Sándor, Z., M. Wedel. 2001. Designing conjoint choice experiments using managers' prior beliefs. Journal of Marketing Research. 38 430-444.

Sándor, Z., M. Wedel. 2002. Profile construction in experimental choice designs for mixed logit models. Marketing Science. 21 455-475.

Sándor, Z., M. Wedel. 2005. Heterogeneous conjoint choice designs. Journal of Marketing Research. 42 210-218.

Toubia, O., J. R. Hauser, D. I. Simester. 2004. Polyhedral methods for adaptive choicebased conjoint analysis. Journal of Marketing Research. 41 116-131.

Train, K. 2003. Discrete Choice Methods with Simulation. Cambridge University Press.

Vermeulen, B., P. Goos, M. Vandebroek. 2008. Models and optimal designs for conjoint choice experiments including a no-choice option. International Journal of Research in Marketing. 25 94-103. 
Yu, J., P. Goos, M. Vandebroek. 2008a. Efficient conjoint choice designs in the presence of respondent heterogeneity. Marketing Science. Forthcoming.

Yu, J., Goos, P., Vandebroek, M., 2008b. A comparison of different Bayesian design criteria for constructing efficient conjoint choice experiments. Research report. 